\title{
Effect of Acid Doping on Junction Characteristics of ITO/ Polyaniline/N719/Ag Diode
}

\author{
MUHAMMAD REZA, ${ }^{1}$ FRY VONI STEKY, ${ }^{1}$ and VEINARDI SUENDO $\mathbb{C}^{1,2,3}$ \\ 1.-Division of Inorganic and Physical Chemistry, Faculty of Mathematics and Natural Sciences, \\ Institut Teknologi Bandung, Bandung, Indonesia. 2.-Research Center for Nanosciences and \\ Nanotechnology, Institut Teknologi Bandung, Jl. Ganesha No. 10, Bandung 40132, Indonesia. \\ 3.—e-mail: vsuendo@chem.itb.ac.id
}

In this paper, we demonstrate the fabrication and electrical characterization of a heterojunction Schottky diode between polyaniline (PANI) and a ruthenium-based organic semiconductor (N719). In this system, PANI behaves as an organic $p$-type conducting polymer while N719 acts as an $n$-type semiconductor. The fabrication was carried out using different methods to deposit each component: solution casting for PANI, spray coating for N719, and screen-printing for silver paste. The PANI film was doped by soaking it in $\mathrm{HCl}$ solutions of different concentrations to form emeraldine salt, i.e., a conductive type of PANI. Electrical characterizations of PANI and the diode were performed using conductivity and current density-voltage $(J-V)$ measurements. The maximum conductivity of PANI was obtained at $3.18 \times 10^{-2} \mathrm{~S} / \mathrm{cm}$ using an $\mathrm{HCl}$ concentration of $1 \mathrm{M}$. The fabricated diode exhibited a low Schottky barrier $\left(\Phi_{\mathrm{B}}=0.48 \mathrm{eV}\right)$ and rectifying behavior $(\gamma \sim 9)$ with moderate ideality factor $(\eta \sim 8)$. Acid doping of PANI caused better diode performance and an increase in current density by four orders of magnitude.

Key words: Heterojunction Schottky diode, polyaniline, emeraldine salt, electrical conductivity, Schottky barrier height, acid doping

\section{INTRODUCTION}

Conducting polymers have attracted great interest in electronic and optoelectronic applications. Polyaniline (PANI) is one of the most intensively studied conducting polymers, which has attracted considerable attention during the last decade due to its flexibility, cost effective chemical synthesis, easy chemical modification, and good environmental stability. ${ }^{1-3}$ PANI film is easy to deposit on many substrates (glass or metals) using different methods, such as dip coating, ${ }^{4}$ spin coating, ${ }^{5}$ emulsion polymerization, ${ }^{6}$ Langmuir-Blodgett technique, ${ }^{7}$ electrochemical deposition ${ }^{8}$ and thermal evaporation. ${ }^{9}$ Several acids (both organic and inorganic) have been used as doping agents to enhance

(Received September 9, 2019; accepted December 13, 2019; published online January 2, 2020) electrical conductivity of PANI, including hydrochloric acid (HCl) ${ }^{10}$ acetic acid, ${ }^{11}$ camphor sulfonic acid, ${ }^{12}$ and even amino acid. ${ }^{13}$ PANI is considered a $p$-type organic semiconductor because it has radical cation species (i.e. polarons), which serve as hole transport materials in electronic devices. ${ }^{14,15}$

A number of research papers have demonstrated the fabrication and junction characteristics of indium tin oxide (ITO)/PANI/metal diode systems. ${ }^{16-19}$ ITO coated glass is usually used as an electron injection contact, while PANI is a low work function polymer that enables hole injection to the electrode. This structure produces a Schottky-type diode with a relatively high barrier height $\left(\Phi_{\mathrm{B}}\right)$. In order to reduce $\Phi_{\mathrm{B}}$, several efforts have been carried out through the organic-inorganic hybrid system. ${ }^{20,21}$ This system combines the properties of inorganic and organic materials for synergistic applications. 
In this article, we investigate the effect of acid doping on junction characteristic of a ITO/PANI/ N719/silver (Ag) heterojunction diode. N719 is a well-known $n$-type semiconductor which is used as donor layer in our diode system. Different concentrations of $\mathrm{HCl}$ were used as acid doping agents on PANI. Fabrication of the diode was performed layerby-layer using different methods. We expect the optimum $\mathrm{HCl}$ concentration and addition of N719 layer will produce a diode with low $\Phi_{\mathrm{B}}$.

\section{EXPERIMENTAL}

\section{Materials}

Aniline, acetone, and graphite were purchased from Sigma-Aldrich. Hydrochloric acid ( $\mathrm{HCl}$ ), ammonium hydroxide $\left(\mathrm{NH}_{4} \mathrm{OH}\right), \mathrm{N}$-methyl pyrrolidone (NMP) and ammonium persulfate (APS) were purchased from Merck. Ruthenium complex N719 was purchased from Synthex Technologies, Poland. Silver paste (Elecolit 340) was purchased from Eleco-Produits, France. ITO glass $\left(R_{\mathrm{sh}}<11 \Omega / \mathrm{sq}\right)$ was purchased from Zhuhai Kaivo Optoelectronic Technology, China. All reagents were analytical grade and used without any further purification.

\section{Synthesis of PANI Emeraldine Salt (PANI ES)}

PANI ES was synthesized using a rapid mixing method. ${ }^{10}$ Two solutions were initially prepared: (1) $3.64 \mathrm{~mL}$ of aniline was dissolved in $100 \mathrm{~mL}$ of $\mathrm{HCl}$ $1 \mathrm{M}$, and (2) $11.42 \mathrm{~g}$ of APS was dissolved in $100 \mathrm{~mL}$ of demineralized water. These two solutions were transferred to a cooling bath $\left(T=0^{\circ} \mathrm{C}\right)$ for $1 \mathrm{~h}$ before mixed together and kept in the same cooling bath for $24 \mathrm{~h}$. The reaction mixture was then filtered, rinsed with $\mathrm{HCl}$ and acetone repeatedly, and dried under dynamic vacuum for $24 \mathrm{~h}$.

\section{Synthesis of PANI Emeraldine Base (PANI EB)}

PANI EB was obtained from the dedoping process of PANI ES in a basic condition. The synthesized PANI ES was dispersed in a solution of $\mathrm{NH}_{4} \mathrm{OH} 1 \mathrm{M}$ for $3 \mathrm{~h}$ under constant stirring. The resulting purplish powder was collected, rinsed with demineralized water, and dried in vacuum oven for $24 \mathrm{~h}$.

\section{Fabrication of Diode}

PANI EB (1\% weight) was dissolved in NMP under constant stirring overnight. This PANI solution was deposited on a $2 \times 2.5 \mathrm{~cm}$ ITO glass substrate, which was previously cleaned with demineralized water and isopropyl alcohol, using a solution casting method and dried at $150^{\circ} \mathrm{C}$. The PANI EB film was then soaked for $15 \mathrm{~min}$ in $\mathrm{HCl}$ solutions of various concentrations to form PANI ES. N719 dye solution in ethanol $\left(5 \times 10^{-4} \mathrm{M}\right)$ was sprayed on the PANI films using an airbrush at $100^{\circ} \mathrm{C}$. As an electrical contact, silver paste was deposited using screen printing and heated at $100^{\circ} \mathrm{C}$. Figure 1 shows the schematic illustration of the PANI/N719 diode fabrication process.

\section{Characterization}

FTIR spectra of PANI samples were obtained by Bruker Alpha FTIR-ATR spectrometer with a resolution of $4 \mathrm{~cm}^{-1}$. An LCR meter Agilent E4980A was used to determine the electrical conductivity of the PANI samples in the form of pellets with diameter of $1.2 \mathrm{~cm}$ and thickness of $0.3 \mathrm{~cm}$. Current-voltage $(I-V)$ measurements of diode were performed using an $I-V$ meter Keithley 2400 .

\section{RESULTS AND DISCUSSION}

\section{Structure and Electronic Properties of PANI}

Figure 2a shows FTIR spectra of PANI EB and several PANI ES doped with various $\mathrm{HCl}$ concentrations. It is observed that the PANI EB and PANI ES spectra produce similar absorption peak patterns, with slight shifts in the wavenumber (10$30 \mathrm{~cm}^{-1}$ ). The main characteristic peaks of PANI are associated with $\mathrm{C}=\mathrm{C}$ stretching from quinoid rings $\left(1559 \mathrm{~cm}^{-1}\right), \mathrm{C}=\mathrm{C}$ from benzenoid rings $\left(1483 \mathrm{~cm}^{-1}\right), \mathrm{C}-\mathrm{N}\left(1306 \mathrm{~cm}^{-1}\right), \mathrm{C}-\mathrm{N}^{+}$in polaronic structure $\left(1244 \mathrm{~cm}^{-1}\right), \mathrm{Q}=\mathrm{NH}^{+}-\mathrm{B}$ and $\mathrm{B}-\mathrm{NH}^{+}-\mathrm{B}$ $\left(1140 \mathrm{~cm}^{-1}\right){ }^{22}$ The last peak at $820 \mathrm{~cm}^{-1}$ is associated with the out-of-plane bending vibration mode of $\mathrm{C}-\mathrm{H} .{ }^{22}$ We observed changes in FTIR spectra with the increase of $\mathrm{HCl}$ concentration as doping agent. Absorbance at $1244 \mathrm{~cm}^{-1}$ increases as $\mathrm{HCl}$ concentration increases. This corresponds to the formation of PANI samples with higher conductivity, indicated by the increase of polaron species (C$\mathrm{N}^{+}$) numbers.

As revealed by Fig. 2b, we observed some changes in conductivity profile for each PANI sample. It appears that the conductivity of PANI EB (without $\mathrm{HCl}$ as doping agent) tends to increase as a function of frequency, which is characteristic of insulating materials. This profile remained unchanged when we used an $\mathrm{HCl}$ concentration of $0.25 \mathrm{M}$. As $\mathrm{HCl}$ concentration increased $(0.50$ and $0.75 \mathrm{M})$, the conductivity profile slightly changed at low frequency, but tended to decrease and maintain its profile at high frequency. Significant change was observed when we used an $\mathrm{HCl}$ concentration of 1.00 M. The conductivity tended to decrease as a function of frequency, which is characteristic of conducting/metallic materials. This happens when metals are placed under an AC (alternating current) electric field. At high frequency, electrons of metal will move back and forward faster, and they will scatter each other more often, resulting in the inhibition of charge transfer. It can be concluded that the synthesized PANI ES had metallic characteristics while the PANI EB had the characteristics of an insulator. ${ }^{23,24}$

Table I presents the conductivity of PANI ES with various concentrations of $\mathrm{HCl}$ at a frequency of 


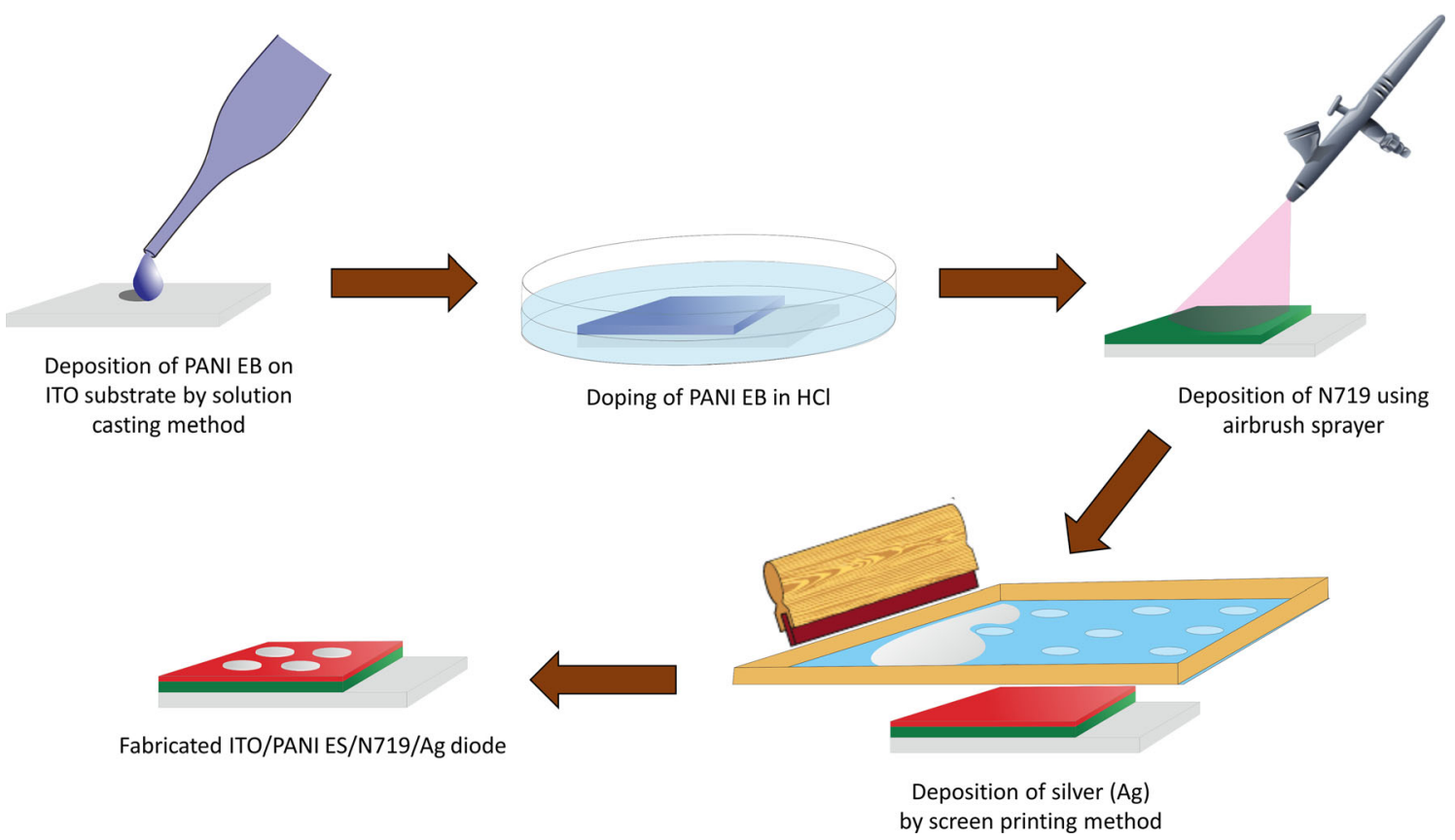

Fig. 1. Schematic illustration of the diode fabrication process. The fabrication was carried out as follows: solution casting of PANI EB onto ITO substrate followed by doping process in $\mathrm{HCl}$ solution, spray coating of $\mathrm{N} 719$ dye, and screen-printing of silver paste as an electrical contact.
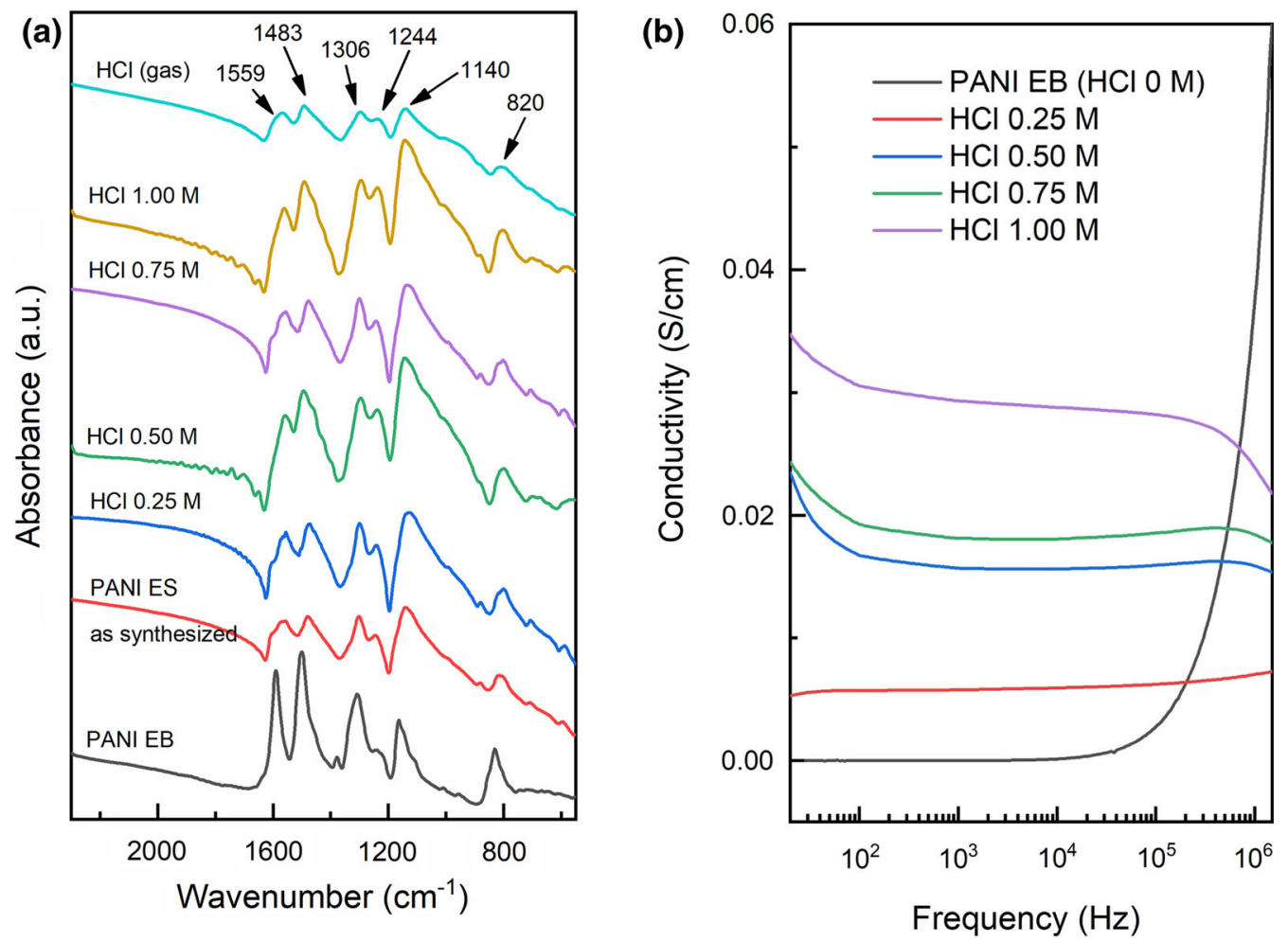

Fig. 2. (a) FTIR spectra and (b) frequency response of electrical conductivity profile of PANI samples doped with various $\mathrm{HCl}$ concentrations.

$50 \mathrm{~Hz}$. As the $\mathrm{HCl}$ concentration increased, the conductivity of PANI at $50 \mathrm{~Hz}$ also increased and reached a maximum at $3.18 \times 10^{-2} \mathrm{~S} / \mathrm{cm}$ with the $\mathrm{HCl}$ concentration of $1 \mathrm{M}$. We observed a similar trend between conductivity and the ratio of absorbance at $1244-1308 \mathrm{~cm}^{-1}$ of PANI ES as a function of $\mathrm{HCl}$ concentration (Fig. 3). This ratio represents the number of polaron species $\left(\mathrm{C}-\mathrm{N}^{+}\right)$ relative to secondary amine $(\mathrm{C}-\mathrm{N})$ in PANI rings. The increase of polaron species, along with the 
Table I. Absorbance ratio and electrical conductivity at $50 \mathrm{~Hz}$ of synthesized PANI doped with various $\mathrm{HCl}$ concentrations

\begin{tabular}{|c|c|c|c|}
\hline No. & [HCl] $(\mathbf{M})$ & $\mathbf{A}_{1244} / \mathbf{A}_{1306}$ & Conductivity at $50 \mathrm{~Hz}(\mathrm{~S} / \mathrm{cm})$ \\
\hline 1 & 0 & 0.594 & $8.21 \times 10^{-6}$ \\
\hline 2 & 0.25 & 0.846 & $5.69 \times 10^{-3}$ \\
\hline 3 & 0.50 & 0.901 & $1.82 \times 10^{-2}$ \\
\hline 4 & 0.75 & 0.922 & $2.07 \times 10^{-2}$ \\
\hline 5 & 1.00 & 0.930 & $3.18 \times 10^{-2}$ \\
\hline
\end{tabular}

Both absorbance ratio and electrical conductivity show similar trends against $\mathrm{HCl}$ concentration

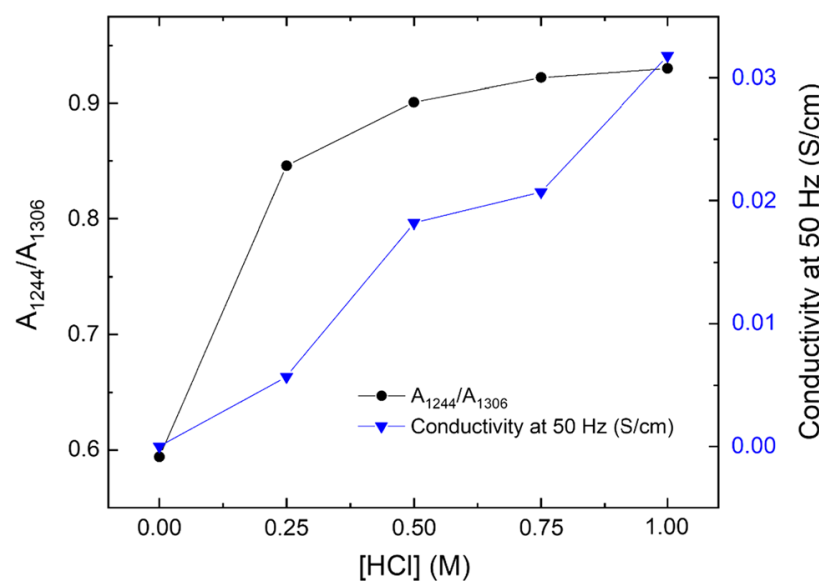

Fig. 3. Relationship between electrical conductivity of PANI at $50 \mathrm{~Hz}$ and $\mathrm{HCl}$ concentration as dopant associated with the amount of polaron species. Absorbance ratio at $1244-1308 \mathrm{~cm}^{-1}$ represents the amount of polaron species $\left(\mathrm{C}-\mathrm{N}^{+}\right)$relative to secondary amine $(\mathrm{C}-\mathrm{N})$ in PANI.

increase of $\mathrm{HCl}$ concentration, leads to the increase of PANI ES conductivity.

\section{Electronic Characteristics of Diodes}

Figure $4 \mathrm{a}$ and $\mathrm{b}$ shows the current density-voltage $(J-V)$ curve and the semilogarithmic plot of the $J-V$ curve of diodes composed by PANI doped with various $\mathrm{HCl}$ concentrations. The $J-V$ curves were slightly nonlinear, and there existed asymmetry with respect to the polarity of $V$, indicating rectification behavior. It can be seen that the resulting current density increases as concentrations of $\mathrm{HCl}$ increases. The current density increases by four orders as acid doping on PANI is performed, which is due to the increase in the number of polaron species. Moreover, the forward current density begins to increase exponentially at lower threshold voltages as $\mathrm{HCl}$ concentration increases $(1.25 \mathrm{~V}$ at $\mathrm{HCl}$ concentrations of $0.25 \mathrm{M}$ and $0.50 \mathrm{~V}$ at $1 \mathrm{M}$ ). This shows that polaron species is responsible as a load carrier (hole) which facilitates current flow in this system. ${ }^{25}$

The rectifying $J-V$ behavior of Schottky barrier devices is usually assumed to follow the standard thermionic emission (TE) theory for conduction across the junction. ${ }^{26,27}$ In this theory, the current is assumed to be controlled only by the transfer of carriers across the interface of the PANI/N719. The current-voltage relationship for the Schottky barrier device is given as a Shockley equation $\mathrm{as}^{28}$

$$
J=J_{0}\left[\exp \left(\frac{q V}{\eta k T}\right)-1\right]
$$

where $J_{O}$ is reverse saturation current density, ${ }^{29} q$ is the electronic charge, $k$ is the Boltzmann constant, and $T$ is temperature in degrees Kelvin. ${ }^{30}$ From Eq. (1), the value of $\eta$ is calculated from the slope of the straight-line region of the forward bias $\ln (J)$ versus $V$ plot $\mathrm{as}^{28}$

$$
\eta=\frac{q}{k T} \ln \left[\frac{\mathrm{d} V}{\mathrm{~d}(\ln J)}\right] .
$$

The value of $J_{0}$ is extracted from the straight-line intercept of the forward bias $\ln (J)$ versus $V$ plot at zero bias from the expression ${ }^{26}$

$$
J_{0}=A^{*} T^{2} \exp \left(-\frac{q \Phi_{\mathrm{B}}}{k T}\right),
$$

where $A^{*}$ is the effective Richardson constant taken as $120 \mathrm{~A} /\left(\mathrm{cm}^{2} \mathrm{~K}^{2}\right)$ and $\Phi_{\mathrm{B}}$ is the apparent barrier height at zero bias. $\Phi_{\mathrm{B}}$ can be obtained by rewriting Eq. (3) $\mathrm{as}^{26}$

$$
\Phi_{\mathrm{B}}=\frac{k T}{q} \ln \left(\frac{A^{*} T^{2}}{J_{0}}\right)
$$

Table II summarizes the diode characteristics for all fabricated PANI diodes in this work.

The obtained barrier height $\left(\Phi_{\mathrm{B}}\right)$ for all diodes is in the range of $0.48-0.54 \mathrm{eV}$. This value is smaller than the PANI diode system and PANI-CdS diode. ${ }^{18,31}$ This value is also smaller than the standard value of $\Phi_{\mathrm{B}}$ of a Schottky-type diode where direct contact between the metal and the semiconductor layer occurs. In general, $\Phi_{\mathrm{B}}$ tends to decrease with increasing $\mathrm{HCl}$ concentration, which indicates that the polaron species facilitates the injection 

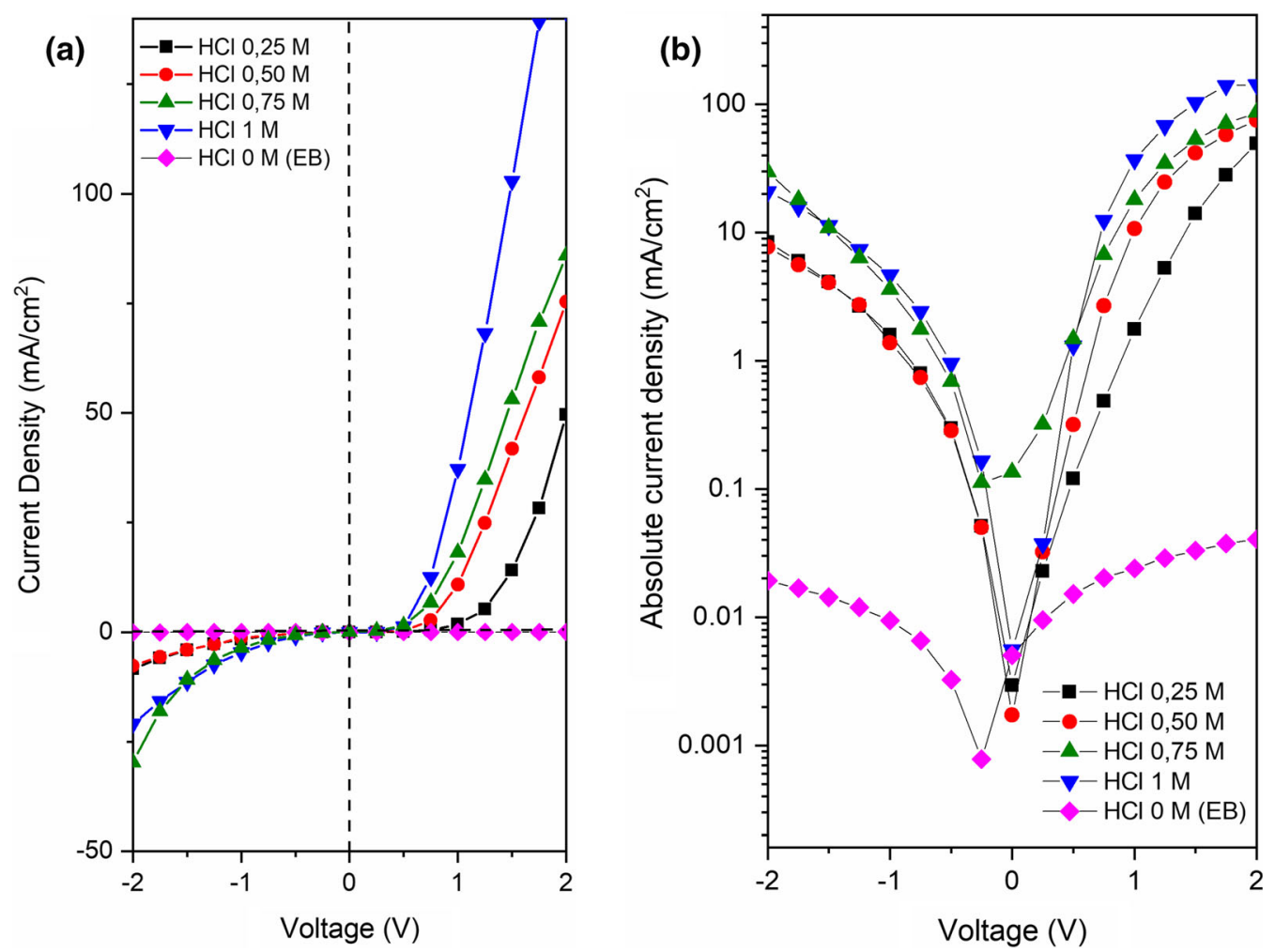

Fig. 4. (a) $J-V$ curve and (b) semilogarithmic plot of respective $J-V$ curve of diodes composed by PANI doped with various $\mathrm{HCl}$ concentrations. The obtained current density $(\mathcal{J}$ increases as a function of $\mathrm{HCl}$ conecntration.

Table II. Electrical characteristics of diodes composed by PANI doped with various HCl concentration which shows a linear relationship between each parameter [threshold voltage, rectification ratio $(\gamma)$, ideality factor $(\eta)$, and barrier height $\left.\left(\Phi_{B}\right)\right]$ and $H C l$ concentration as dopant

\begin{tabular}{|c|c|c|c|c|c|}
\hline No. & {$[\mathrm{HCl}](\mathrm{M})$} & Threshold voltage (V) & $\gamma @ 1.25 \mathrm{~V}$ & $\eta$ & $\Phi_{B}(e V)$ \\
\hline 1 & 0 & 7.25 & 2.42 & 58.89 & 0.540 \\
\hline 2 & 0.25 & 1.25 & 1.98 & 10.20 & 0.520 \\
\hline 3 & 0.50 & 1.00 & 3.93 & 11.94 & 0.488 \\
\hline 4 & 0.75 & 0.50 & 5.47 & 17.71 & 0.501 \\
\hline 5 & 1 & 0.50 & 9.22 & 7.71 & 0.498 \\
\hline
\end{tabular}

process of the hole from the anode to the active layer of the semiconductor. However, the obtained value of $\eta$ is still high if we compare it to the standard $\eta$ of Schottky diodes (around 4 or 5 ). ${ }^{32}$ The best $\eta$ is obtained at $\mathrm{HCl}$ concentration of $1 \mathrm{M}(\eta=7.71)$. Some factors contribure to $\eta$, such as the presence of interface layers, charge recombination, and migration from electrode material. One of the most likely factors is the presence of aggregates (Fig. 5) that appear during the fabrication process, resulting in the presence of several different transport mechanisms. ${ }^{33}$

The charge recombination in the depletion region causes current flow negative bias. Based on the Schottky barrier theory, ${ }^{34}$ the work function of the metal must be smaller than that of the $p$-type semiconductor so that the rectification barrier will be formed at the interface. Not all diodes have shown the good rectification ability, indicated by their rectification ratio $(\gamma)$. The obtained $\gamma$ is still on the order of $10^{1}$. This indicates that there is still a leakage of current in negative bias, which is probably caused by an imperfect fabrication process, especially in the deposition process of the active layers

\section{CONCLUSION}

We fabricated a heterojunction diode consisting of polyaniline emeraldine salt (PANI ES) and ruthenium complex N719 as a active $p$ - and $n$ - semiconducting materials, respectively. Low work function metal silver was used as a contact. Thermionic emission theory was used to determine the junction characteristics of the diodes. Acid doping using $\mathrm{HCl}$ solution caused higher conductivity of PANI, indicated by an increased number of polaron species. 


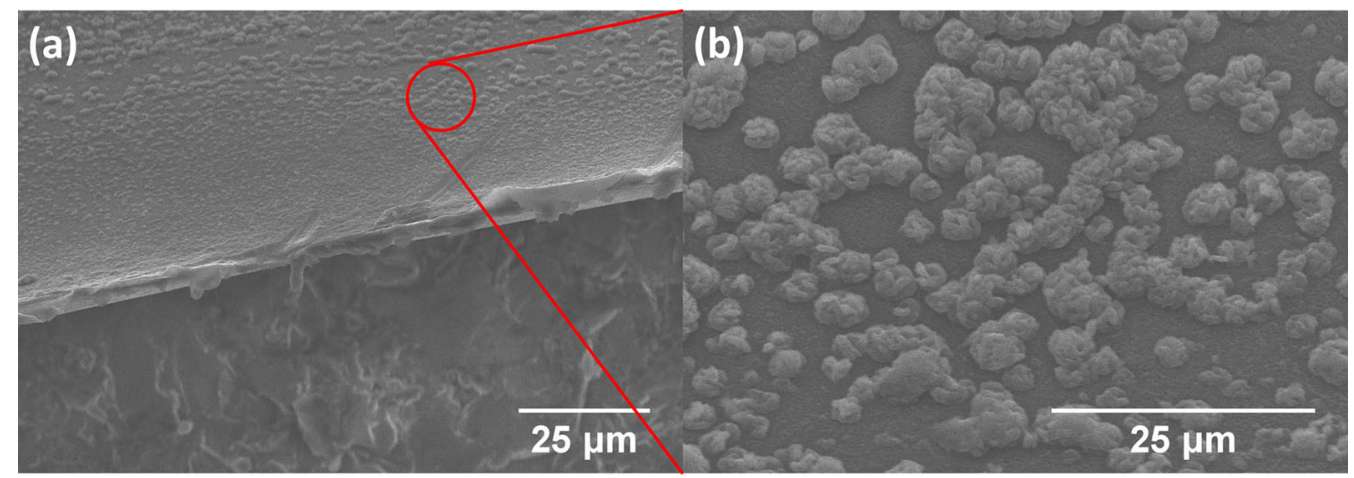

Fig. 5. SEM image of active layer of diode at (a) $1000 \times$ and (b) $5000 \times$ magnification, which shows the presence of aggregates. Aggregates mainly affect the ideality factor $(\eta)$ and rectification ratio $(\gamma)$ of diodes due to different transport mechanisms.

$\mathrm{HCl}$ concentration of $1 \mathrm{M}$ resulted in the maximum conductivity of PANI of $3.18 \times 10^{-2} \mathrm{~S} / \mathrm{cm}$. Acid doping also affected the junction characteristic of the fabricated diodes, significantly decreasing the threshold voltage and increasing the rectification ratio. The $J-V$ measurement showed that ITO/ PANI ES $(1 \mathrm{M}) / \mathrm{N} 719 / \mathrm{Ag}$ structure behaves like a Schottky barrier type diode with rectification ratio of 9.22 at $1.25 \mathrm{~V}$, ideality factor of 7.71 , and barrier height of $0.498 \mathrm{eV}$.

\section{ACKNOWLEDGMENTS}

This work was financially funded by ITB Research Grant 2018 through ITB Research and Innovation Program 2018. M. Reza acknowledges Ministry of Education and Culture of Indonesia for scholarship through Beasiswa Unggulan (contract number: 54192/A1.4/LL/2017).

\section{REFERENCES}

1. J. Huang, Pure Appl. Chem. 78, 15 (2006).

2. R. Wang, L. Huang, and X. Tian, J. Phys. Chem. C 116, 13120 (2012).

3. J. Stejskal, P. Kratochvil, and A.D. Jenkins, Polym 37, 367 (1996).

4. M. Deniz and H. Deligöz, Colloids Surf. A 563, 206 (2019).

5. M.R. Choi, S.H. Woo, T.H. Han, K.G. Lim, S.Y. Min, T.W. Lee, W.M. Yun, O.K. Kwon, C.E. Park, K.D. Kim, H.K. Shin, M.S. Kim, T. Noh, J.H. Park, K.H. Shin, and J. Jang, ChemSusChem 4, 363 (2011).

6. Z. Mo, W. Qui, X.C. Yang, and J. Yan, J. Pol. Res. 16, 39 (2009).

7. J.H. Cheung, A.F. Fou, and M.F. Rubner, Thin Solid Films 84, 985 (1994).

8. D. Chinn, J. DuBow, J. Li, J. Janata, and M. Josowicz, Chem. Mater. 7, 1510 (1995).

9. N.E. Agbor, M.C. Petty, and A.P. Monkman, Sens. Actuators B Chem. 28, 173 (1995).

10. J.C. Chiang and A.G. Macdiarmid, Synth. Met. 13, 193 (1986).

11. D.W. Hatchett, M. Josowicz, and J. Janata, J. Phys. Chem. B 103, 10992 (1999).

12. A.G. MacDiarmid and A.J. Epstein, Synth. Met. 69, 85 (1995).

13. Y. Noskov, A. Sorochinsky, V. Kukhar, and A. Pud, ACS Omega 4, 7400 (2019).
14. D. Yaohua and M. Shaoli, Electrochim. Acta 36, 2015 (1991)

15. S. Ahn, M.H. Park, S.H. Jeong, Y.H. Kim, J. Park, S. Kim, H. Kim, H. Cho, C. Wolf, M. Pei, H. Yang, and T.W. Lee, Adv. Funct. Mater. 1807535, 1 (2018).

16. C. Li, Y. Wang, M. Wan, and S. Li, Synth. Met. 39, 91 (1990).

17. S.A. Chen, K.R. Chuang, C.I. Chao, and H.T. Lee, Synth. Met. 82, 207 (1996).

18. P.J. Saikia and P.C. Sarmah, Mat. Sci. Appl. 2, 1022 (2011).

19. N.S. Singh, L. Kumar, A. Kumar, S. Vaisakh, S.D. Singh, K. Sisodiya, S. Srivastava, M. Kansal, S. Rawat, and T.A. Singh, Tanya, Anita. Mater. Sci. Semicond. Process. 60, 29 (2017).

20. S.K. Dey, S. Baglari, and D. Sarka, Indian J. Phys. 90, 29 (2016).

21. J.M. Ghushe, S.M. Giripunje, and S.B. Kondawar, J. Phys. Sci. 28, 99 (2017).

22. M. Trchova and J. Stejskal, Pure Appl. Chem. 83, 1803 (2011).

23. Y. Wei, X. Tang, and Y. Sun, J. Pol. Sci. 27, 2385 (1989).

24. R. Singh, V. Arora, R.P. Tandon, S. Chandra, and A. Mansingh, J. Mater. Sci. 33, 2067 (1998).

25. S. Chen and Y. Fang, Synth. Met. 60, 215 (1993).

26. H. Tomozawa, F. Braun, S. Phillps, A.J. Heeger, and H. Kroemer, Synth. Met. 22, 63 (1987).

27. E.H. Rhoderick, Metal-Semiconductor Contacts (Monographs in Electrical and Electronic Engineering (Oxford: Clarendon Press, 1978), p. 96.

28. S.M. Sze, Physics of Semiconductor Devices (New York: Wiley, 1971).

29. S. Boughdachi, Y. Badali, Y. Azizian-kalandaragh, and S. Altindal, J. Elect. Mat. (2018). https://doi.org/10.1007/s116 64-018-6593-y.

30. N.S. Singh, L. Kumar, A. Kumar, S. Vaisakh, S.D. Singh, K. Sisodiya, S. Srivastava, M. Kansal, S. Rawat, and ThA Singh, Tanya, Anita. Mat. Sci. Semicond. Process. 60, 29 (2017).

31. S.K. Dey, S. Baglari, and D. Sarkar, Indian. J Phys. 90, 29 (2016).

32. L. Pereira, Organic Light-Emitting Diodes: The Use of RareEarth and Transition Metals (Boca Raton: Pan Stanford Publishing, 2012), pp. 48-93.

33. S. Angappane, N. Rajeev Kimi, T.S. Natarajan, G. Rangarajan, and B. Wessling, Thin Solid Films 417, 202 (2002).

34. J.C.W. Chien, Polyacetylene: Chemistry, Physics, and Material Science (Orlando: Academic Press, 1984), p. 581.

Publisher's Note Springer Nature remains neutral with regard to jurisdictional claims in published maps and institutional affiliations. 